CALT-68-1959

\title{
Semileptonic Decays of Heavy Tetraquarks
}

\author{
Chi-Keung Chow \\ California Institute of Technology, Pasadena, CA 91125
}

(April 29, 2018)

\begin{abstract}
There exist heavy tetraquark states $Q Q \bar{q} \bar{q}$ in the heavy quark limit. These states are stable with respect to strong interactions and hence must decay weakly. It is shown that the semileptonic decay depends on a single IsgurWise form factor, which can be expressed in term of the Isgur-Wise form factors which govern the semileptonic decays of the $Q q q$ and $Q Q q$ baryons.
\end{abstract}


The possibility of the existence of exotics, i.e, hadrons with properties incompatible with a $q \bar{q}$ or $q q q$ description, is an important issue in QCD. In particular, the existence of tetraquark (or di-meson) states has been proposed by Jaffe [1] and since then investigated by many other groups [2 25$]$.

The case of binding two heavy quarks to two light antiquarks to form a heavy tetraquark $Q Q \bar{q} \bar{q}$ is especially interesting. The two heavy quarks are bound by the short range Coulumbic chromoelectric attraction in the $3 \otimes 3 \rightarrow \overline{3}$ channel. The bound state will have binding energy of order $E \sim \alpha_{s}^{2}\left(m_{Q}\right) m_{Q}$. When $m_{Q}$ is so large such that $E \gg \Lambda_{\mathrm{QCD}}$, the large binding energy forbids the dissociation $Q Q \bar{q} \bar{q} \rightarrow Q \bar{q}+Q \bar{q}$. Moreover, the light degrees of freedom cannot resolve the closely bound $Q Q$ system, which has size of order $\left(m_{Q} \alpha_{s}\left(m_{Q}\right)\right)^{-1} \ll \Lambda_{\mathrm{QCD}}^{-1}$. This results in bound states that have "brown mucks" similar to those of $\bar{\Lambda}_{Q}$ states, with $Q Q$ playing the role of the heavy antiquark. Hence the stability of $\bar{\Lambda}_{Q}$ implies that $Q Q \bar{q} \bar{q}$ is also safe from decaying through $Q Q \bar{q} \bar{q} \rightarrow Q Q q+\bar{q} \bar{q} \bar{q}$. As the result, $Q Q \bar{q} \bar{q}$ is stable with respect to strong interactions and must decay weakly.

Since the "brown mucks" of the ground state tetraquarks are spinless, the spins of the tetraquarks are just given by the spins of the heavy quarks. As the result, the ground states are degenerate spin $S=0$ or 1 tetraquarks. We will denote the tetraquarks by $|T(p, S, m)\rangle$ where $m$ is the $z$-component of the spin. The normalizations of these states are given by

$$
\left\langle T\left(p^{\prime}, S^{\prime}, m^{\prime}\right) \mid T(p, S, m)\right\rangle=16 \pi^{3} \delta_{S S^{\prime}} \delta_{m m^{\prime}} \delta^{3}\left(\mathbf{p}-\mathbf{p}^{\prime}\right),
$$

such that, when the masses of the tetraquarks go to infinity, the states are still well defined.

Since the two heavy quarks are bound by a Coulumbic potential, we can represent the heavy degrees of freedom as

$$
\left|Q_{a} Q_{b}(v, S, m)\right\rangle=\int d^{3} \mathbf{p}\left|Q_{a}\left(v_{a}, s_{a}\right)\right\rangle \otimes\left|Q_{b}\left(v_{b}, s_{b}\right)\right\rangle \otimes \psi(B ; \mathbf{p})\left(\frac{1}{2}, s_{a} ; \frac{1}{2}, s_{b} \mid S, m\right) .
$$

The Clebsch-Gordon coefficient describes the spin structure of the tetraquark, and $\mathbf{p}=$ $m_{a}\left(\mathbf{v}-\mathbf{v}_{\mathbf{a}}\right)=-m_{b}\left(\mathbf{v}-\mathbf{v}_{\mathbf{b}}\right)$ is the relative momentum between the heavy quarks. $\psi(B ; \mathbf{p})$ is the ground state Coulumbic wavefunction in momemtum space 


$$
\psi(B ; \mathbf{p})=\frac{4 B^{5 / 2}}{\left(\mathbf{p}^{2}+B^{2}\right)^{2}},
$$

with $B=\mu_{a b} \alpha_{s}\left(\mu_{a b}\right)$ the reciprocal of the Bohr radius. Together with the spinless light degrees of freedom $|\phi(v)\rangle$, we get the decomposition

$$
\begin{aligned}
\left|T_{a b}(v, S, m)\right\rangle & =\left|Q_{a} Q_{b} \bar{q} \bar{q}(v, S, m)\right\rangle \\
& =\int d^{3} \mathbf{p}\left|Q_{a}\left(v_{a}, s_{a}\right)\right\rangle \otimes\left|Q_{b}\left(v_{b}, s_{b}\right)\right\rangle \otimes \psi(B ; \mathbf{p}) \otimes|\phi(v)\rangle\left(\frac{1}{2}, s_{a} ; \frac{1}{2}, s_{b} \mid S, m\right) .
\end{aligned}
$$

We define the Isgur-Wise form factor $\tilde{\eta}_{a b c}(w)$ of the semileptonic $T_{a b} \rightarrow T_{a c}$ decay by

$$
\begin{aligned}
& \left\langle T_{a c}\left(v^{\prime}, S^{\prime}, m^{\prime}\right)\left|\bar{Q}_{c} \Gamma Q_{b}\right| T_{a b}(v, S, m)\right\rangle \\
& \quad=\tilde{\eta}_{a b c}(w) \delta_{s_{a} s_{a}^{\prime}} \bar{u}_{c}\left(v^{\prime}, s_{c}^{\prime}\right) \Gamma u_{b}\left(v, s_{b}\right)\left(\frac{1}{2}, s_{a} ; \frac{1}{2}, s_{b} \mid S, m\right)\left(\frac{1}{2}, s_{a}^{\prime} ; \frac{1}{2}, s_{c}^{\prime} \mid S^{\prime}, m^{\prime}\right),
\end{aligned}
$$

where $w=v \cdot v^{\prime}$. The form factor $\tilde{\eta}_{a b c}(w)$ contains contributions from both perturbative QCD, which describes the attraction between the two heavy quarks, and non-perturbative QCD, which accounts for the interaction of the $Q Q$ system with the light degrees of freedom.

By Eq. (4), the matrix element can also be decomposed as

$$
\begin{aligned}
\left\langle T_{a c}\left(v^{\prime}, S^{\prime}, m^{\prime}\right)\left|\bar{Q}_{c} \Gamma Q_{b}\right| T_{a b}(v, S, m)\right\rangle & =\int d^{3} \mathbf{p}^{\prime} \int d^{3} \mathbf{p} \psi\left(C ; \mathbf{p}^{\prime}\right) \psi(B ; \mathbf{p})\left\langle\phi\left(v^{\prime}\right) \mid \phi(v)\right\rangle\left\langle Q_{a}\left(v_{a}^{\prime}, s_{a}^{\prime}\right) \mid Q_{a}\left(v_{a}, s_{a}\right)\right\rangle \\
& \left\langle Q_{c}\left(v_{c}^{\prime}, s_{c}^{\prime}\right)\left|\bar{Q}_{c} \Gamma Q_{b}\right| Q_{b}\left(v_{b}, s_{b}\right)\right\rangle\left(\frac{1}{2}, s_{a} ; \frac{1}{2}, s_{b} \mid S, m\right)\left(\frac{1}{2}, s_{a}^{\prime} ; \frac{1}{2}, s_{c}^{\prime} \mid S^{\prime}, m^{\prime}\right),
\end{aligned}
$$

where analogously $\mathbf{p}^{\prime}=m_{a}\left(\mathbf{v}^{\prime}-\mathbf{v}_{\mathbf{a}}^{\prime}\right)=-m_{c}\left(\mathbf{v}^{\prime}-\mathbf{v}_{\mathbf{c}}^{\prime}\right)$ and $C=\mu_{a c} \alpha_{s}\left(\mu_{a c}\right)$.

In order the evaluate $\left\langle\phi\left(v^{\prime}\right) \mid \phi(v)\right\rangle$, recall that

$$
\left|\bar{\Lambda}_{Q}(v, s)\right\rangle=|\bar{Q}(v, s)\rangle \otimes|\phi(v)\rangle
$$

Then

$$
\begin{aligned}
\left\langle\bar{\Lambda}_{c}\left(v^{\prime}, s^{\prime}\right)\left|\bar{Q}_{b} \Gamma Q_{c}\right| \bar{\Lambda}_{b}(v, s)\right\rangle & =\left\langle\phi\left(v^{\prime}\right) \mid \phi(v)\right\rangle\left\langle\bar{Q}_{c}\left(v^{\prime}, s^{\prime}\right)\left|\bar{Q}_{b} \Gamma Q_{c}\right| \bar{Q}_{b}(v, s)\right\rangle \\
& =\left\langle\phi\left(v^{\prime}\right) \mid \phi(v)\right\rangle \bar{v}_{c}\left(v^{\prime}, s^{\prime}\right) \Gamma v_{b}(v, s)
\end{aligned}
$$

The Isgur-Wise form factor $\eta(w)$ of $Q q q$ baryons are defined by [6] 


$$
\left\langle\bar{\Lambda}_{c}\left(v^{\prime}, s^{\prime}\right)\left|\bar{Q}_{b} \Gamma Q_{c}\right| \bar{\Lambda}_{b}(v, s)\right\rangle=\eta(w) \bar{v}_{c}\left(v^{\prime}, s^{\prime}\right) \Gamma v_{b}(v, s) .
$$

Hence we get

$$
\eta(w)=\left\langle\phi\left(v^{\prime}\right) \mid \phi(v)\right\rangle
$$

On the other hand, we can also evaluate the $Q_{b} \rightarrow Q_{c}$ matrix element.

$$
\left\langle Q_{c}\left(v_{c}^{\prime}, s_{c}^{\prime}\right)\left|\bar{Q}_{c} \Gamma Q_{b}\right| Q_{b}\left(v_{b}, s_{b}\right)\right\rangle=\bar{u}_{c}\left(v_{c}^{\prime}, s_{c}^{\prime}\right) \Gamma u_{b}\left(v_{b}, s_{b}\right)
$$

However, since both $\mathbf{v}_{\mathbf{b}}-\mathbf{v}=\mathbf{p} / m_{b}$ and $\mathbf{v}_{\mathbf{c}}^{\prime}-\mathbf{v}^{\prime}=\mathbf{p}^{\prime} / m_{c}$ are small quantities of the order of $\Lambda_{\mathrm{QCD}} / m_{Q}$, we can write

$$
\left\langle Q_{c}\left(v_{c}^{\prime}, s_{c}^{\prime}\right)\left|\bar{Q}_{c} \Gamma Q_{b}\right| Q_{b}\left(v_{b}, s_{b}\right)\right\rangle \sim \bar{u}_{c}\left(v^{\prime}, s_{c}^{\prime}\right) \Gamma u_{b}\left(v, s_{b}\right)+O\left(\Lambda_{\mathrm{QCD}} / m_{Q}\right) .
$$

The subleading terms will be neglected in our discussion.

Lastly, making use of the fact that

$$
\left\langle Q_{a}\left(v_{a}^{\prime}\right) \mid Q_{a}\left(v_{a}\right)\right\rangle=\delta^{3}\left(m_{a} \mathbf{v}_{\mathbf{a}}^{\prime}-m_{a} \mathbf{v}_{\mathbf{a}}\right)=\delta^{3}\left(\mathbf{p}^{\prime}-\mathbf{p}-\mathbf{q}\right),
$$

with $\mathbf{q}=m_{a}\left(\mathbf{v}^{\prime}-\mathbf{v}\right)$, one of the integrals can be evaluated. Then we get

$$
\begin{array}{r}
\left\langle T_{a c}\left(v^{\prime}, S^{\prime}, m^{\prime}\right)\left|\bar{Q}_{c} \Gamma Q_{b}\right| T_{a b}(v, S, m)\right\rangle=\int d^{3} \mathbf{p} \psi(C ; \mathbf{p}+\mathbf{q}) \psi(B ; \mathbf{p}) \delta_{s_{a} s_{a}^{\prime}} \bar{u}_{c}\left(v^{\prime}, s_{c}^{\prime}\right) \Gamma u_{b}\left(v, s_{b}\right) \\
\eta(w)\left(\frac{1}{2}, s_{a} ; \frac{1}{2}, s_{b} \mid S, m\right)\left(\frac{1}{2}, s_{a}^{\prime} ; \frac{1}{2}, s_{c}^{\prime} \mid S^{\prime}, m^{\prime}\right) .
\end{array}
$$

To see the significance of the integral, consider the semileptonic decay $Q_{a} Q_{b} q \rightarrow Q_{a} Q_{c} q$ which is described by a single Isgur-Wise form factor $\eta_{a b c}(w)$ [10. The "brown muck" of a $Q Q q$ baryon is similar to that of a heavy meson $\bar{Q} q$, with the two closely bound heavy quarks playing the role of the antiquark. As the result, the analysis goes exactly as above except that the overlap of the light degrees of freedom gives the mesonic Isgur-Wise form factor $\frac{(w+1)}{2} \xi(w)$ instead of $\eta(w)$. Setting the slow varying $\frac{(w+1)}{2} \xi(w)$ to unity, we have

$$
\left\langle Q_{a} Q_{c} q\left(v^{\prime}\right)\left|\bar{Q}_{c} \Gamma Q_{b}\right| Q_{a} Q_{b} q(v)\right\rangle=\int d^{3} \mathbf{p} \psi(C ; \mathbf{p}+\mathbf{q}) \psi(B ; \mathbf{p}) \bar{u}_{c}\left(v^{\prime}\right) \Gamma u_{b}(v) .
$$

Hence, according to the definition in Ref. [10], we conclude that 


$$
\eta_{a b c}(w)=\eta_{a b c}\left(1-\frac{\mathbf{q}^{2}}{2 m_{a}^{2}}\right)=\int d^{3} \mathbf{p} \psi(C ; \mathbf{p}+\mathbf{q}) \psi(B ; \mathbf{p}) .
$$

Hence we finally obtain

$$
\begin{aligned}
& \left\langle T_{a c}\left(v^{\prime}, S^{\prime}, m^{\prime}\right)\left|\bar{Q}_{c} \Gamma Q_{b}\right| T_{a b}(v, S, m)\right\rangle \\
& \quad=\eta(w) \eta_{a b c}(w) \delta_{s_{a} s_{a}^{\prime}} \bar{u}_{c}\left(v^{\prime}, s_{c}^{\prime}\right) \Gamma u_{b}\left(v, s_{b}\right)\left(\frac{1}{2}, s_{a} ; \frac{1}{2}, s_{b} \mid S, m\right)\left(\frac{1}{2}, s_{a}^{\prime} ; \frac{1}{2}, s_{c}^{\prime} \mid S^{\prime}, m^{\prime}\right) .
\end{aligned}
$$

Comparing with Eq. (5), we get the main result of this paper.

$$
\tilde{\eta}_{a b c}(w)=\eta(w) \eta_{a b c}(w)
$$

$\eta_{a b c}(w)$ describes the perturbative attraction between the two heavy quarks, while $\eta(w)$ accounts for the non-perturbative interaction between the heavy quarks and the "brown muck."

It is in order to discuss the $w$-dependences of various form factors involved in our discussion. The meson Isgur-Wise form factor $\xi(w)$ is a slow varying function as its slope at the point of zero recoil is of the order of unity. On the other hand, $\psi(B, \mathbf{p})$ has a short range of the order of $B \sim m_{Q} \alpha_{s}\left(m_{Q}\right)$. Hence, near the point of zero recoil, the slope $\eta_{a b c}(w)$ is of the order of $\alpha_{s}^{-2}\left(m_{Q}\right)$. In the heavy quark limit, this slope is large $\left(\alpha_{s}^{-2}\left(m_{b}\right) \sim 22.5\right)$ and the $w$-dependence of $\eta_{a b c}(w)$ does overwhelm that of $\xi(w)$. This justifies the neglect of the $\xi(w)$ factor in Eq. (15). On the other hand, we expect the baryon Isgur-Wise form factor $\eta(w)$ to be slow varying in the real world. In some models, however, $\eta(w)$ has strong $w$-dependence. For example, in the large $N_{c}$ limit [11, 12], the slope at the point of zero recoil is of the order of $N_{c}^{3 / 2}$. Hence we keep $\eta(w)$ in our final expression Eq. (18) for generality.

By Luke's Theorem [13], $\eta(w)$ is normalized to unity in the heavy quark limit.

$$
\eta(1)=1
$$

No analogous statement exists for $\eta_{a b c}(w)$, the analytical form of which is given in Ref. [10. Indeed it can be seen that

$$
\eta_{a b c}(1)=\left(\frac{2 \sqrt{B C}}{B+C}\right)^{3}
$$


which is not equal to unity unless $B=C$, i.e, $m_{b}=m_{c}$. This is very different from the normalization of $\eta(w)$, which holds regardless of the size of $m_{b}-m_{c}$ as long as both $m_{b}$ and $m_{c} \gg \Lambda_{\mathrm{QCD}}$.

In conclusion we found that the semileptonic decays of heavy tetraquarks are described by a single Isgur-Wise form factor $\tilde{\eta}_{a b c}(w)$, which can be factorized into two pieces. The piece due to non-perturbative QCD is just the Isgur-Wise form factor $\eta(w)$ for $Q q q \rightarrow Q q q$ decays, while the perturbative piece is $\eta_{a b c}(w)$, the Isgur-Wise form factor for $Q Q q \rightarrow Q Q q$ decays.

The discussion above is expected to hold when $\alpha_{s}^{2}\left(m_{Q}\right) m_{Q} \gg \Lambda_{\mathrm{QCD}}$. In the real world, since the top quark does not live long enough to form hadrons, we just got two "hadronizable" heavy quarks, the $b$-quark and the $c$-quark. The assumption above, however, holds for neither of them, and our results cannot be applied directly. Still it is possible that the picture above is at least qualitatively correct and can serve as the starting point of quantitative investigations of the heavy tetraquark systems by including the effects of $1 / m_{Q}$ corrections.

This work was supported in part by the U.S. Dept. of Energy under Grant No. DEFG03-92-ER 40701. 


\section{REFERENCES}

[1] R.J. Jaffe, Phys. Rev. D15 267 (1977).

[2] M.J. Savage and M.B. Wise, Phys. Lett. B248 177 (1990).

[3] J.M. Richard, Nucl. Phys. Proc. Suppl. B21 254 (1991).

[4] J.M. Richard, in "Quark Cluster Dynamics" ed. K. Goeke, P. Kroll and H.R. Petry (1992).

[5] A.V. Manohar and M.B. Wise, Nucl. Phys. B399 17 (1993).

[6] N. Isgur and M.B. Wise, Nucl. Phys. B348 276 (1991).

[7] H. Georgi, Nucl. Phys. B348 293 (1991).

[8] T. Mannel, W. Roberts and Z. Ryzak, Nucl. Phys. B355 38 (1991).

[9] F. Hussain, J.G. Korner, M. Kramer and G. Thompson, Z. Phy C51 321 (1991).

[10] M.J. White and M.J. Savage, Phys. Lett. B271 410 (1991).

[11] E. Jenkins, A.V. Manohar and M.B. Wise, Nucl. Phys. B396 38 (1991).

[12] C.K. Chow, CALT-68-1946, accepted by Phys. Rev. D (1994).

[13] M. Luke, Phys. Lett. B252 447 (1990). 